Revue internationale P.M.E.

\title{
Gestion des relations banques-PME
}

Les apports de la théorie du contrat social

The management of banks-SME relationships

The contributions of the social contract theory

Gestión de las relaciones bancos-PYMES

Las contribuciones de la teoría del contrato social

\section{Laure Ambroise, Isabelle Maque et Isabelle Prim-Allaz}

Volume 26, numéro 2, 2013

URI : https://id.erudit.org/iderudit/1024320ar

DOI : https://doi.org/10.7202/1024320ar

Aller au sommaire du numéro

Éditeur(s)

Editions EMS - In Quarto SARL

ISSN

0776-5436 (imprimé)

1918-9699 (numérique)

Découvrir la revue

Citer cet article

Ambroise, L., Maque, I. \& Prim-Allaz, I. (2013). Gestion des relations banques-PME : les apports de la théorie du contrat social. Revue internationale P.M.E., 26(2), 37-58. https://doi.org/10.7202/1024320ar
Résumé de l'article

Les approches des échanges, par les théories des coûts de transaction des contrats incomplets et de la dépendance ou de la théorie des conventions, ont proposé des conceptualisations riches, mais souvent jugées incomplètes. Une alternative intéressante proposée par Ian Macneil, la théorie du contrat social est désormais largement répandue en gestion. Au fil du temps, cette théorie s'est imposée comme une clé de lecture pertinente dans l'analyse des relations et de leur gouvernance, notamment au travers des relations clients/fournisseurs. Elle permet de mieux comprendre ces relations offrant ainsi aux entreprises la possibilité d'améliorer leurs relations clients, mais aussi leurs relations internes. Si la théorie du contrat social remet globalement en cause l'idée selon laquelle une relation peut être totalement décrite, formalisée, anticipée, voire contractualisée, elle propose également un cadre permettant de catégoriser et d'analyser les différentes dimensions et facettes d'une relation. Cette recherche vise à préciser comment les normes contractuelles permettent de compléter et de prolonger les théories classiques de l'échange. Selon cette conception, l'ensemble des interactions est alors réellement appréhendé selon une approche relationnelle et holiste. L'analyse assortie de deux études complémentaires (l'une avec une approche marketing et l'autre en finance organisationnelle) sur les relations entre les PME et leurs banques a été réalisée. Les résultats montrent la pertinence de la théorie du contrat social en sciences de gestion, notamment comme cadre de compréhension des mécanismes de gouvernance des échanges bancaires. Ils mettent également en exergue le rôle de la gestion de la relation dans la création de valeur. L’intérêt opérationnel de cette approche de ce cadre théorique est ainsi démontré. 


\title{
Gestion des relations banques-PME : les apports de la théorie du contrat social
}

\begin{abstract}
Laure AMBROISE
Laure AMBROISE est maître de conférences en marketing à l'Université de Saint-Étienne, rattachée au centre de recherche COACTIS - EA 4161. Le point commun de ses recherches est l'analyse des relations selon des angles différents. Elle a contribué à plusieurs recherches appréhendant la gestion de la relation client comme levier de croissance pour les PME. Elle a également publié des articles sur la relation marque consommateur, notamment dans Recherche et Applications en Marketing.

Université de Lyon/Saint-Étienne - Laboratoire COACTIS

6, rue Basse des Rives

42023 SAINT-ETIENNE CEDEX 2

laure.ambroise@univ-st-etienne.fr
\end{abstract}

Isabelle MAQUE

Isabelle MAQUE est agrégée du secondaire en économie et gestion, docteure en sciences de gestion, maître de conférences en finance, rattachée au centre de recherche en gestion - CEREGE - EA 1722 - à l'IAE de l'université de Poitiers (France). Ses recherches portent principalement sur les relations banques-entreprises, les relations banques-PME, les échanges relationnels et le financement des PME.

Université de Poitiers, IAE - Laboratoire CEREGE 20, rue Guillaume-VII le Troubadour BP 639 86022 POITIERS CEDEX imaque@iae.univ-poitiers.fr

\section{Isabelle PRIM-ALLAZ ${ }^{1}$}

Isabelle PRIM-ALLAZ est agrégée du secondaire en économie et gestion, docteure en sciences de gestion, maître de conférences en marketing, rattachée au centre de recherche COACTIS - EA 4161 - Université de Lyon 2 (France). Ses recherches portent principalement sur les relations entre les banques et les PME (échanges relationnels versus transactionnels), la gestion de la relation client et la croissance des entreprises. Elle a, entre autres, publié dans Recherche et Applications en Marketing et Décisions Marketing.

Université de Lyon/ Lyon 2 - Laboratoire COACTIS
ISH - 14, avenue Berthelot
69363 LYON CEDEX
Isabelle.Prim-Allaz@univ-lyon2.fr

1 En hommage au professeur Jean Perrien. 


\begin{abstract}
RÉSUMÉ
Les approches des échanges, par les théories des coûts de transaction des contrats incomplets et de la dépendance ou de la théorie des conventions, ont proposé des conceptualisations riches, mais souvent jugées incomplètes. Une alternative intéressante proposée par Ian Macneil, la théorie du contrat social est désormais largement répandue en gestion. Au fil du temps, cette théorie sest imposée comme une clé de lecture pertinente dans lanalyse des relations et de leur gouvernance, notamment au travers des relations clients/fournisseurs. Elle permet de mieux comprendre ces relations offrant ainsi aux entreprises la possibilité d'améliorer leurs relations clients, mais aussi leurs relations internes. Si la théorie du contrat social remet globalement en cause l'idée selon laquelle une relation peut être totalement décrite, formalisée, anticipée, voire contractualisée, elle propose également un cadre permettant de catégoriser et d'analyser les différentes dimensions et facettes d'une relation. Cette recherche vise à préciser comment les normes contractuelles permettent de compléter et de prolonger les théories classiques de léchange. Selon cette conception, l'ensemble des interactions est alors réellement appréhendé selon une approche relationnelle et holiste. L'analyse assortie de deux études complémentaires (l'une avec une approche marketing et l'autre en finance organisationnelle) sur les relations entre les PME et leurs banques a été réalisée. Les résultats montrent la pertinence de la théorie du contrat social en sciences de gestion, notamment comme cadre de compréhension des mécanismes de gouvernance des échanges bancaires. Ils mettent également en exergue le rôle de la gestion de la relation dans la création de valeur. L'intérêt opérationnel de cette approche de ce cadre théorique est ainsi démontré.
\end{abstract}

MOTS CLÉS

Macneil, Contrat social, Banque, PME, Échange, Relation, Transaction

\title{
The management of banks-SME relationships : the contributions of the social contract theory
}

\begin{abstract}
Transaction costs and incomplete contracts convention theories propose different interesting conceptualizations of exchanges but are often considered incomplete. The social contract theory is an interesting alternative proposed by Ian Macneil that is now widely used in management. This theory has emerged over time as a key character to a better understanding and analysis of relationships and their governance, particularly for customer/supplier relationships. It helps to better understand these relationships and thus offer businesses the opportunity to enhance their relationships with their customers but also internal relationships. If the social contract theory calls into question the whole idea that a relationship can be fully described, anticipated, formalized or contractualized, it also provides a framework to categorize and analyze the different dimensions and facets of a relationship. This research aims at clarifying how contractual norms can complement and extend the classical theories of exchange. According to this framework, all interactions are then genuinely apprehended in a relational and holistic approach. Two studies (one in marketing and the other in organisational finance) on the relationships between SMEs and their banks are proposed in order to illustrate the framework relevance. Results show the relevance of the social contract theory in business management, especially as a framework for understanding the governance mechanisms of SME-bank relationships. The role of relationship management is also highlighted as being important in creating value. The operational advantage of Macneil's framework is therefore demonstrated.
\end{abstract}

KEY WORDS

Macneil, Social contract, Bank, SME, Exchange, Relationship, Transaction 


\title{
Gestión de las relaciones bancos-PYMES : las contribuciones de la teoria del contrato social
}

\begin{abstract}
RESUMEN
Las teorías de costes de transacción y de convenios y de contratos incompletos conllevan diferentes conceptualizaciones relevantes de los intercambios pero que a menudo han sido juzgadas como incompletas. Una alternativa interesante es la propuesta de Ian Macneil, es decir, la teoría del contrato social que es en la actualidad ampliamente difundida en gestión. Con el tiempo, esta teoría se ha convertido en la clave para una mejor comprensión y análisis de las relaciones y su gobernanza, sobre todo para las relaciones cliente-proveedor. Esta teoría permite comprender mejor estas relaciones ofreciendo así a las empresas la posibilidad de mejorar sus relaciones con los clientes pero también sus relaciones internas. Si la teoría del contrato social pone en cuestión la idea de que una relación puede ser totalmente descrita, formalizada, anticipada e incluso contractualizada, propone también un marco que permite categorizar y analizar las diferentes dimensiones y facetas de una relación. Esta investigación tiene por objeto establecer cómo las normas contractuales pueden complementar y ampliar las teorías clásicas de intercambio. De acuerdo con este marco, todas las interacciones son comprendidas según un enfoque relacional y holístico. Con el fin de ilustrar la relevancia de dicho marcho han sido efectuados dos estudios (uno desde el marketing y el otro desde las finanzas corporativas) sobre las relaciones entre las PYMES y sus bancos. Los resultados muestran la relevancia de la teoría del contrato social en la gestión empresarial, especialmente como marco de comprensión de los mecanismos de gobernanza de los intercambios bancarios. El papel de la gestión de las relaciones también ha sido destacado como importante para la creación de valor. Además, la ventaja operativa del marco de Macneil ha sido también demostrada.
\end{abstract}

Palabras Clave

Macneil, Contrato social, Banco, PYME, Intercambio, Relación, Transacción

\section{INTRODUCTION}

Les dernières décennies ont été l'occasion d'une profonde transformation des entreprises, tant en ce qui concerne leur organisation, leur management et leur stratégie. Comme l'explique l'Institut de l'entreprise dans son rapport annuel de 2010, " plusieurs phénomènes ont ainsi conduit à la reconfiguration du processus de création de valeur et des modèles économiques qui y sont associés : émergence du client au centre des organisations, éclatement géographique de la chaîne de valeur permise par la libéralisation des échanges commerciaux avec les pays du Sud, apparition du low cost, montée en puissance de léconomie numérique, etc. ». Face à ces nouveaux enjeux, la gestion des relations au sens large est une des problématiques managériales majeures que rencontrent les dirigeants d’entreprise ; ainsi, cette thématique est devenue au fil des années une thématique essentielle en gestion, notamment en marketing où la gestion de la relation clients est au cœur des réflexions stratégiques et des processus organisationnels depuis les années 1990.

Pour autant, si la relation est un concept central dans l'interaction de l'entreprise avec son environnement, il n’existe pas de théorie permettant d'appréhender et de qualifier de façon globale 
les relations que l’entreprise entretient avec ses partenaires. Bien évidemment, les échanges intra et inter-organisationnels ont fait l'objet de nombreuses attentions, notamment de la part des économistes au travers des théories des coûts de transaction, des contrats incomplets et de la dépendance ou de la théorie des conventions. Toutefois, ces approches ont souvent été jugées incomplètes, car elles ne permettent pas d'appréhender les dimensions irrationnelles et informelles des relations réelles. Un auteur a pourtant élaboré et proposé une théorie alternative, la théorie du contrat social, dont l'objet est justement d'apprécier les aspects économiques et sociaux d'une relation : ainsi, Ian Macneil publie en 1980 un ouvrage intitulé The new social contract : an inquiry into modern contractual relations.

Originellement développée en sciences juridiques, la théorie du contrat social remet globalement en cause l'idée selon laquelle une relation peut être totalement décrite, formalisée, anticipée, voire contractualisée ; l'auteur propose toutefois un cadre permettant de catégoriser et d'analyser les différentes dimensions et facettes d'une relation. Il identifie ainsi des normes dites « normes contractuelles » qui permettent d'apprécier une relation dans une perspective plutôt transactionnelle ou plutôt relationnelle (Macneil, 1980).

Lobjet de cet article est d'étudier dans quelle mesure le cadre théorique proposé par Macneil peut constituer une clé de lecture pertinente dans l'analyse des relations et de leur gestion au sein des entreprises. Ainsi, nous pensons que la théorie du contrat social peut accroitre la compréhension des mécanismes particuliers de gestion des relations quentretient l'entreprise avec ses différents partenaires, tant dans une perspective intra que inter-organisationnelle. Si certaines recherches ont d'ores et déjà mis en exergue l'intérêt de cette théorie en gestion, et tout particulièrement en marketing pour appréhender les relations clients/fournisseurs (Young, Gilbert et McIntyre, 1996), il s’avère que le cadre proposé constitue une approche intégrative au-delà des clivages disciplinaires ou organisationnels. Il offre ainsi aux entreprises la possibilité de disposer d'un outil global d’analyse, mais aussi de mieux comprendre et gérer les relations qu'elles entretiennent avec leurs partenaires.

Compte tenu du rôle, crucial et sans cesse rappelé, des banques dans le financement des PME, cette recherche se focalise de façon spécifique sur les rapports qu'entretiennent celles-ci avec leurs banques et étudie dans quelle mesure le cadre de référence proposé par Macneil permet d'améliorer la gestion de ces relations. L’objectif est d'apprécier dans quelle mesure une gestion avertie et volontaire des relations d'une PME avec ses partenaires bancaires influence la dynamique de l'organisation ainsi que son efficience. En effet, plusieurs auteurs soutiennent qu'une meilleure compréhension des mécanismes particuliers de coopération participe aux mécanismes de création de valeur (Charreaux et Wirtz, 2006) ; la notion de la valeur est entendue dans une perspective partenariale, c'est-à-dire la valeur sécrétée par la firme dans ses transactions avec l'ensemble de ses partenaires (Charreaux et Desbrières, 1998), et plus spécifiquement ici, ses partenaires bancaires.

Dans une première partie, les fondamentaux de la théorie du contrat social sont présentés et les principales limites discutées. Lobjectif est de préciser comment les normes contractuelles permettent de compléter et de prolonger les théories classiques de l'échange. Selon cette conception, l'ensemble des interactions est alors réellement appréhendé selon une approche relationnelle et holiste. Dans un second temps, la méthodologie retenue est présentée. En effet, afin d'appréhender sous différents angles d'analyse les relations PME-banques et in fine de mieux les comprendre, nous avons opté dans le cadre de cette recherche pour une analyse 
assortie de données. Une troisième partie montre comment la théorie du contrat social permet de comprendre la nature des relations que les PME entretiennent avec leurs partenaires bancaires et comment ces relations peuvent ainsi être gérées dans l'intérêt réciproque d'une plus grande création de valeur. La conclusion met en perspective les apports de la recherche menée ainsi que les limites et les voies de recherches futures.

\section{LA THÉORIE DU CONTRAT SOCIAL DE MACNEIL}

L’objectif principal de cette étude étant l'opérationnalisation de la théorie du contrat social pour aider les PME et les banques à mieux gérer leurs relations, nous allons dans un premier temps rappeler les fondements de cette théorie en précisant ses intérêts et limites pour les sciences de gestion. En effet, la théorie du contrat social se différencie des théories classiques et néoclassiques par son appréhension de la continuité temporelle des échanges, étudiant les échanges au-delà des transactions ponctuelles. Elle repose également sur l'idée que les échanges se construisent autour de normes ${ }^{2}$ et sur la distinction des normes transactionnelles et relationnelles. Cependant, malgré sa richesse, cette théorie doit faire face à un certain nombre de limites présentées à la fin de cette section.

\subsection{Les approches classiques de l'échange}

La littérature sur les échanges propose classiquement deux grandes familles d'approches : les approches transactionnelles et relationnelles.

Les approches transactionnelles sont notamment représentées par les théories économiques classique et néoclassique. L'approche classique (microéconomique) met l'accent sur des opérations ponctuelles généralement prévues et décrites dans un contrat. Léchange est déconnecté de toute relation passée ou future. Les approches néoclassiques prolongent cette analyse en considérant des achats répétés, mais qui restent cependant indépendants les uns des autres (Williamson, 1985 ; Gomez, 1994). Dans ces types de contrat, la gouvernance de l'échange ne reconnait pas d'interactions dynamiques des parties prenantes et la relation est considérée comme une externalité, interprétée d'un point de vue microéconomique et utilitariste. Les parties se mettent d'accord sur la forme d'échange économique la plus efficace et font la différence entre la gouvernance par les marchés (prix) et les hiérarchies (autorité légale).

Les approches relationnelles considèrent, quant à elles, des échanges répétés à caractère relationnel, c'est-à-dire des échanges ayant une orientation à long terme délibérée. Elles mettent en exergue le rôle des phénomènes d'interaction dans les échanges et s'intéressent à l'ensemble de la relation entre les partenaires et non pas seulement à un accord spécifique (Durif et Perrien, 2008). Dans ce cadre de référence, l'unité d'analyse n'est plus la transaction, mais la relation. La transaction s'inscrit dès lors dans le contexte historique

2 Terme ayant une portée sémantique très précise pour Macneil que nous développerons ultérieurement. 
d'une relation plus globale. Le contrat est relationnel dans le sens où les parties sont incapables de prédéterminer, de façon exhaustive, les termes importants du contrat en obligations complètes et bien définies (Macneil, 2000 ; Paulin et Ferguson, 2010). Selon cette approche, la notion de contrat est prise dans un sens très large comprenant un ensemble de règles informelles, normes et pratiques. Les approches relationnelles se différencient des approches transactionnelles également en abordant la relation et sa coordination comme un phénomène bilatéral où des adaptations et des ajustements ont lieu en permanence. Ces ajustements reposent sur des attentes et des normes de comportement partagées qui peuvent différer largement d'un contexte à un autre (Thibaut et Kelley, 1959). Ainsi, les approches relationnelles placent l'interaction entre les individus au cœur des mécanismes des échanges et font de la relation un élément vivant et difficilement prévisible, notamment $\mathrm{du}$ fait de contingences futures. La Figure 1 reprend les principales différences des deux approches.

FiguRE 1. LES DIFFÉRENTES FORMES D’ÉCHANGE ET LEURS CARACTÉRISTIQUES

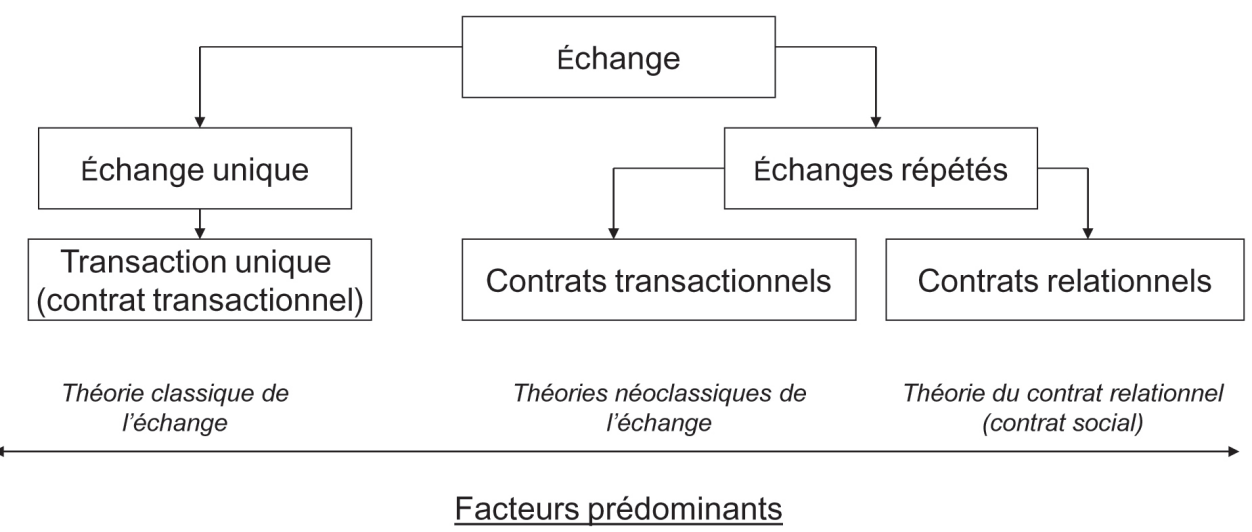

Prédominance des facteurs prix et qualité intrinsèque des produits/ services d'interaction* : confiance, réciprocité

Transaction

Unité d'analyse

Transaction

Relation (ensemble de transactions et d'interactions)

Perspectives temporelles

Ponctuelle (pas d'interaction future)

Orientation à long terme " délibérée " (forte probabilité d'interactions futures)

* Le terme interaction est à prendre au sens de « rencontre entre des individus » et non pas au sens statistique du terme.

Typologie retenue d’après les travaux de Macneil (1980) et Williamson (1985).

L'importance des échanges justifie et explique que les organisations cherchent à mieux les formaliser et les contrôler. Selon Macaulay (1963), les entreprises fonctionnent fréquemment 
avec des normes informelles et sur des pratiques " habituelles " au secteur d'activité dans lequel elles opèrent. Ainsi, il s'avère que les relations intra et inter-organisationnelles se caractérisent par de fortes relations interpersonnelles, une communication relativement intense et des éléments significatifs de satisfaction personnelle non économique. Face à ces constats, l'objet de la présente recherche est dévaluer dans quelle mesure la théorie du contrat social s'adapte à l'approche managériale de l'entreprise et contribue ainsi à améliorer la compréhension des mécanismes d’échanges, de coopérations et de relations des entreprises. Par conséquent, la prochaine section développe les spécificités de la théorie proposée par Macneil.

\subsection{La théorie du contrat social (Macneil, 1980)}

\subsubsection{Présentation générale du CAdre de la théorie du Contrat SOCIAL}

Un des intérêts fondamentaux du cadre d'analyse proposé par Macneil (1980), par rapport aux théories classique et néoclassique de l'échange, est la remise en cause de l'idée que les acteurs sont guidés uniquement par la maximisation du profit. Les parties à l'échange poursuivent un ensemble d'objectifs dont l'un d'eux seulement est la maximisation du profit (Whitford, 1985). Ces théories ne sont alors pas suffisantes pour expliquer la gouvernance des échanges dans nos sociétés complexes parce qu'elles ne permettent pas de prendre en compte la composante relationnelle présente dans tous les échanges à des degrés divers. Cette dernière recouvre lélément temporel où les actions passées ont une influence sur les actions présentes qui, elles-mêmes, auront une influence sur les actions futures. La relation, dans ce contexte, est appréhendée dans sa continuité (Dwyer, Schurr et Oh, 1987 ; Perrien, Paradis et Bantig, 1995), ce qui s'explique par des coûts économiques à la sortie, mais aussi, par des coûts psychologiques plus particulièrement liés aux aspects informels de l'échange. Les acteurs sont des êtres socialisés dont l'identité est en partie constituée par leurs relations et donc leurs interactions (Heide et Wathne, 2006).

Pour caractériser les échanges, Macneil trace un continuum allant du contrat classique au contrat relationnel (Macneil, 1981 ; Paulin et Ferguson, 2010). Dans le contrat classique, la relation est totalement décrite, une chose à la fois est traitée, le consentement et l'efficacité sont mis en avant, le système juridique est le moyen de protection principal et la dimension monétaire est la seule mesure de l'échange. Dans le contrat relationnel, il n’y a pas forcément de lien entre la complétude des clauses contractuelles et la force de la relation. Le défaut de formalisme originel n’empêche pas le développement de normes (non contractuelles) qui finissent par constituer un cadre de référence parfois très complet, mais toujours très souple et évolutif. Ainsi, les relations entre les partenaires et leur coordination reposent sur une gouvernance bilatérale où des ajustements sont discutés et opérés quasi continuellement. Ce cadre est établi avec la volonté de durer, de maintenir la flexibilité et d’inciter à la renégociation. C'est dans la perspective de ce contrat plus informel, que Macneil met en exergue l'importance des « normes » qui le composent. 


\subsubsection{LES NORMES CONTRACTUELLES, RELATIONNELLES ET TRANSACTIONNELLES}

Les normes sont définies comme des types de sentiments et comportements acceptables par les membres d'un système d'échanges, ayant la force d'une obligation ou d'une pression sociale (Macneil, 1978, 1980). Les normes ainsi définies représentent, selon l'auteur, l'ensemble des composantes des échanges, tout au moins l'ensemble des composantes endogènes à la relation.

Afin de formaliser l'aspect informel des relations, Macneil $(1980,1983)$ identifie dix normes ${ }^{3}$, qualifiées de normes contractuelles communes, essentielles à loccurrence de tout échange, quelle que soit sa forme (cf. Tableau 1).

Tableau 1. Composantes des normes contractuelles, D’après Macneil (1980, 1981, 1983)

\begin{tabular}{|c|c|c|c|}
\hline \multicolumn{4}{|l|}{ Normes contractuelles } \\
\hline \multicolumn{2}{|l|}{ Normes relationnelles } & \multicolumn{2}{|c|}{ Normes transactionnelles } \\
\hline Normes & Composantes & Normes & Composantes \\
\hline $\begin{array}{l}\text { Intégrité du rôle } \\
+ \text { communication }\end{array}$ & $\begin{array}{l}\text { Communication } \\
\text { et moyens de } \\
\text { communication } \\
\text { Cohérence } \\
\text { Histoire de la relation }\end{array}$ & Réciprocité & $\begin{array}{l}\text { Amélioration } \\
\text { réciproque de la } \\
\text { situation des acteurs } \\
\text { Division du surplus } \\
\text { équitable }\end{array}$ \\
\hline $\begin{array}{l}\text { Solidarité } \\
\text { (Maintien de la } \\
\text { relation) }\end{array}$ & $\begin{array}{l}\text { Confiance } \\
\text { Continuité / fidélité }\end{array}$ & $\begin{array}{l}\text { Mise en œuvre du } \\
\text { planning }\end{array}$ & $\begin{array}{l}\text { Planification } \\
\text { Ponctualité }\end{array}$ \\
\hline $\begin{array}{l}\text { Flexibilité } \\
\text { (harmonisation des } \\
\text { conflits relationnels) }\end{array}$ & $\begin{array}{l}\text { Adaptabilité } \\
\text { Anticipation }\end{array}$ & $\begin{array}{l}\text { Réalisation des } \\
\text { promesses }\end{array}$ & $\begin{array}{l}\text { Respect des } \\
\text { engagements }\end{array}$ \\
\hline Bien-fondé des moyens & $\begin{array}{l}\text { Procédures } \\
\text { Modes de gouvernance } \\
\text { de la relation }\end{array}$ & $\begin{array}{l}\text { Réparation, confiance, } \\
\text { attentes }\end{array}$ & $\begin{array}{l}\text { Réparation } \\
\text { Confiance }\end{array}$ \\
\hline $\begin{array}{l}\text { Normes supra- } \\
\text { contractuelles }\end{array}$ & $\begin{array}{l}\text { Environnement social } \\
\text { et politique }\end{array}$ & $\begin{array}{l}\text { Création et restriction } \\
\text { de pouvoir }\end{array}$ & $\begin{array}{l}\text { Répartition des } \\
\text { pouvoirs }\end{array}$ \\
\hline
\end{tabular}

Par souci de simplification, nous appelons ici normes transactionnelles l'ensemble des normes non définies par Macneil comme étant relationnelles. Les normes relationnelles permettent une meilleure caractérisation des échanges de nature relationnelle. Les normes transactionnelles sont essentielles à la réalisation de tous les contrats, mais contrairement aux normes

3 En réalité, dans ses travaux initiaux, Macneil (1980) avait identifié neuf normes. Il en rajoute une dixième dans des travaux ultérieurs, le bien-fondé des moyens (Macneil, 1983). 
relationnelles, elles ne jouent pas un rôle majeur dans des contextes relationnels (Fink, Hatten et James, 2010 ; Blois et Ivens 2007).

Ainsi, ces normes permettent de catégoriser, en sous-ensembles homogènes, les déterminants de l'interaction entre les individus. Leur développement repose sur des échanges d'informations, le système ne pouvant bien fonctionner que si le degré d’asymétrie entre les partenaires est tolérable et que la confiance se développe (Lamarque, 2007).

Par ailleurs, l’ensemble des normes de la relation identifiées par Macneil est polymorphe et évolue en fonction du contexte de l'échange. De ce fait, l'analyse du respect ou des déviations par rapport aux normes et l'étude de leur impact sur le mode de comportement des acteurs peut permettre de comprendre le passage de la relation d'une étape de son cycle de vie à l'autre (Perrien, Graf et Colombel, 2005). Chaque déviation représente une menace pour la cohésion et la qualité de la relation, et peut donc être vue comme un facteur potentiel de dégradation de cette relation ou de destruction de valeur (Prim-Allaz, 2000 ; Ambroise, Maque et Prim-Allaz, 2011). En effet, de façon plus générale, plusieurs auteurs soulignent le lien étroit entre la gestion des interactions et la création de valeur (Ford, 1990 ; Caby et Hirigoyen, 2001). Porter et Kramer (2011) mettent notamment en exergue la notion de valeur partagée comme concept fondamental du futur déploiement des organisations, un des leviers de développement de cette valeur étant le renforcement de groupements de partenaires autour de l'entreprise (local cluster development). Dans la lignée de ce précepte, il apparait alors nécessaire de disposer d'un cadre permettant de décrire, comprendre et gérer les relations entre partenaires afin de maximiser la création de valeur partagée.

En résumé et de façon très schématique, la théorie du contrat social permet donc d’appréhender plus spécifiquement l'échange relationnel ; ce dernier se différencie de l'échange transactionnel sur deux points :

- Léchange relationnel a une dimension sociale forte qui s’ajoute à la dimension économique (Dwyer et al., 1987). Cette dimension sociale met l'interaction au cœur de la relation et traduit une " valeur relationnelle » de l'échange ;

- Léchange relationnel a un horizon temporel élargi du fait de l'importance des échanges futurs (Dwyer et al., 1987) et des événements passés (Varadarajan et Rajaratnam, 1986).

Les limites du cadre d’analyse proposé par Macneil sont de différents ordres, conceptuels, mais également méthodologiques. Une première limite est liée au fait que Macneil a été particulièrement prolifique et que sa pensée n’a cessé dévoluer au cours du temps, ce qui pose la question de la stabilité du cadre d’analyse proposé et explique certaines différences entre les auteurs dans la mise en œuvre de ce cadre (Ivens et Blois, 2004) ${ }^{4}$. Macneil (1983) insiste également, à l'instar de nombreuses recherches utilisant ses normes, sur l'importance du contexte sectoriel, historique et culturel, et par conséquent sur la contingence dans la définition des normes. Celle-ci peut également expliquer les différences observées en matière d’opérationnalisation des normes.

4 Par exemple, les travaux antérieurs à 1983 parlent de "mutuality», ceux postérieurs à 1983 de « reciprocity». 
Par ailleurs, la principale limite de l'approche de Macneil demeure avant tout la présentation des normes très abstraites et le fait que l'auteur ne propose jamais d'indications en vue de leur opérationnalisation : il ne fait référence à aucun outil concret permettant de gérer les relations (Durif et Perrien, 2008 ; Blois et Ivens, 2006). Selon Whitford (1985), c'est ce qui explique la lenteur du transfert de cette approche vers d'autres champs de recherche que le droit. Lobjet de ce travail est justement de relier concrètement les normes du cadre théorique aux pratiques de gestion adoptées dans les organisations, et de façon plus spécifique, d'étudier l'intérêt de la théorie du contrat social pour mieux comprendre les relations banques-PME afin d’en améliorer leur gestion du point de vue de l'entreprise sans se limiter à une seule catégorie de déterminants selon l'angle fonctionnel (par exemple, stratégie, finance ou marketing) par lequel on observerait l'entreprise.

\section{MÉTHODOLOGIE : ANALYSE ASSORTIE DE DEUX ÉTUDES DANS LE DOMAINE BANCAIRE}

L'approche méthodologique retenue dans le cadre de cette recherche résulte de la rencontre de deux chercheurs habitués à mobiliser, chacun dans son domaine de compétence, la théorie du contrat social pour analyser et expliquer les relations inter-organisationnelles. Les échanges ont abouti à une convergence de points de vue : la théorie du contrat social peut offrir un cadre d'analyse intégrateur à l'étude, la compréhension et la gestion des relations au sein des entreprises. Cette vision commune est ainsi devenue lobjet de la présente recherche. Pour ce faire, la réutilisation des données, collectées par ces chercheurs lors de précédentes recherches, et leur assortiment, se sont imposés comme une approche méthodologique pertinente pour ce nouvel objet de recherche.

En effet, comme le soulignent Chabaud et Germain (2006), dans l'optique de capitaliser les connaissances empiriques, la réutilisation de résultats et de données initialement collectées dans le cadre d'études conduites de façon indépendante peut être très pertinente et enrichissante. Cette démarche méthodologique est ainsi originale dans la mesure où elle repose sur la ré-exploitation des données collectées par les auteurs eux-mêmes, mais avec un objectif détourné des objectifs initiaux respectifs.

Les différentes disciplines de gestion appréhendent très souvent les relations de façon très parcellaire. La littérature en finance se concentre généralement sur un aspect pragmatique de la relation bancaire qui est le besoin de financement des entreprises. La littérature en marketing, quant à elle, étudie souvent les relations commerciales au sein des établissements bancaires. Le croisement des données collectées au cours des deux recherches a permis d’apprécier dans leur globalité les déterminants de la relation bancaire des PME (points communs) et de montrer que la théorie du contrat social offre un cadre détude complet. Les différences des données croisées (champs disciplinaires et méthodologies) n’ont fait que conforter la pertinence de l'objet de la recherche et des résultats obtenus. 


\title{
ENCADRÉ 1. PrÉSENTATION DES DEUx ÉTUdes UtiliséEs COMME BASE DE L'ANALYSE ASSORTIE
}

\begin{abstract}
La première collecte de données repose sur une démarche à la fois qualitative (étude par entretiens semi-directifs de 13 dyades clients PME/chargés d'affaires - environ 600 pages de retranscription) et quantitative auprès de $300 \mathrm{PME}$ (enquête par questionnaires administrés en face à face auprès de dirigeants de PME ou de directeurs financiers pour les entreprises de taille plus importante). Cette recherche visait à mieux identifier, mesurer et comprendre les déterminants relationnels, notamment le maintien ou non de la relation, avec un regard marketing (Prim-Allaz, 2000).

La seconde collecte de données est de nature strictement qualitative. Il s'agit de 4 études de cas longitudinales permettant la collecte d'informations - entretiens répétés avec les dirigeants et les comptables - sur les relations bancaires de 4 PME durant une période de 2 ans (1 148 pages de retranscription). Ces données qualitatives ont été également croisées avec des informations comptables des entreprises étudiées (bilan, compte de résultats, documents bancaires, etc.). Ancré en finance organisationnelle, l'objet de la recherche était de comprendre comment les entreprises gèrent leurs relations bancaires et les raisons de leur choix (Maque, 2007).
\end{abstract}

Lanalyse assortie des données vise ici à montrer que le cadre théorique des normes contractuelles est suffisamment global et transdisciplinairement robuste pour aider à une meilleure compréhension des relations entre les banques et les PME. La nouvelle analyse des données a reposé sur les points communs et différences des données rassemblées.

1. Les deux recherches menées initialement portaient sur un même domaine d'application : l'analyse des relations entre les banques et les PME;

2. Elles ont également utilisé le même cadre théorique de référence, le contrat social proposé par Macneil, afin détudier, déjà, les relations banques-entreprises dans leur globalité ;

3. Cependant, les études ont été réalisées dans deux champs disciplinaires différents, le marketing et la finance organisationnelle ;

4. Enfin, les données collectées l'ont été selon deux méthodologies différentes (respectivement quantitative et qualitative - cf. Encadré 1 - Prim-Allaz, 2000 ; Maque, 2007)

De façon concrète, les auteurs ont effectué une lecture croisée des résultats et des données initiales avec comme clé d'analyse les thèmes de la gestion des relations et de la création de valeur. Le fait que les auteurs aient un accès privilégié aux données a facilité le travail d'appropriation ; de plus, la vision et l'analyse croisées des données ont renforcé et enrichi les tâches de ré-analyse et de réinterprétation ainsi que la pertinence et la robustesse des résultats (rappelons que les données sont issues de deux champs disciplinaires différents et quelles sont collectées selon deux méthodologies différentes). Les principaux enseignements tirés de ce travail sont à présent déclinés. 


\section{RÉSULTATS}

\subsection{La théorie du contrat relationnel, un cadre de compréhension des mécanismes de gouvernance des échanges bancaires}

Les données utilisées soulignent l'importance de l'ensemble des normes contractuelles dans la gouvernance des échanges bancaires. En effet, il s'avère que les échanges ne sont pas simplement régulés par des mécanismes de marché, et que des caractéristiques de nature "sociale» jouent également un rôle dans la progression de la relation et dans la détermination de son devenir. Les résultats présentés dans les paragraphes suivants mettent en évidence des schémas d'influence différents selon la nature des normes considérées (transactionnelles/relationnelles) et une force variable de cette influence selon les normes.

\subsubsection{LES CHEMINS D'INFLUENCE DES NORMES}

Lanalyse des données montre que le dirigeant de PME va souvent valoriser de façon très importante le fait que le conseiller clientèle de sa banque fasse réellement l'effort de comprendre son domaine d'activité (cf. norme Intégrité $d u$ rôle), qu'il puisse adapter les règles de gestion des comptes en fonction du contexte - ex. problème de trésorerie - (cf. norme Flexibilité), qu'il puisse le soutenir dans des projets capitaux (cf. norme Solidarité). Tous ces comportements du chargé de relation clientèle vont directement impacter la volonté du dirigeant de poursuivre la relation et accroître son propre comportement solidaire vis-à-vis de la banque.

De façon différente, si le dirigeant considère que les contrats ou services proposés par la banque sont pertinents et d'un intérêt mutuel (cf. norme de Réciprocité), que la banque a toujours respecté ses engagements (cf. norme de Réalisation de la promesse) et dans les délais prévus (cf. norme de Mise en ouvre du planning), alors il va globalement considérer qu'il entretient une " bonne relation » avec son établissement bancaire et, de ce fait, va trouver opportun de poursuivre sa relation.

\subsubsection{LA HIÉRARCHISATION DE L'INFLUENCE DES NORMES}

Les données montrent également le rôle différencié de chacune des normes contractuelles dans la gouvernance des échanges bancaires.

Ainsi, les données quantitatives permettent de hiérarchiser l'influence de chacune des normes contractuelles (cf. Figure 2) : la norme qui impacte le plus la volonté de maintenir la relation est celle d'Intégrité du rôle. Ainsi, l'antériorité des échanges a un effet positif sur le désir de poursuivre la relation ; ceci s'explique notamment par une connaissance réciproque des modes de fonctionnement, par des implications dans des projets passés communs qui ont permis de développer la maturité et la satisfaction de la relation. Ces résultats convergent avec ceux issus des études de cas tant pour l'intégrité du rôle du dirigeant que pour l'intégrité du rôle du chargé d’affaires. Ces résultats sont également convergents avec ceux de Durif (2007) qui met en lumière l'importance de la norme Intégrité du rôle dans les relations inter-entreprises, notamment dans le secteur des services financiers. 
Figure 2. Force de LA RELATion entre LeS NORMES DE LA RELATION ET LA DÉCision DE RUPTURE - ÉTUDE QUANTITATIVE EN MARKETING INTER-ORGANISATIONNEL ${ }^{5}$

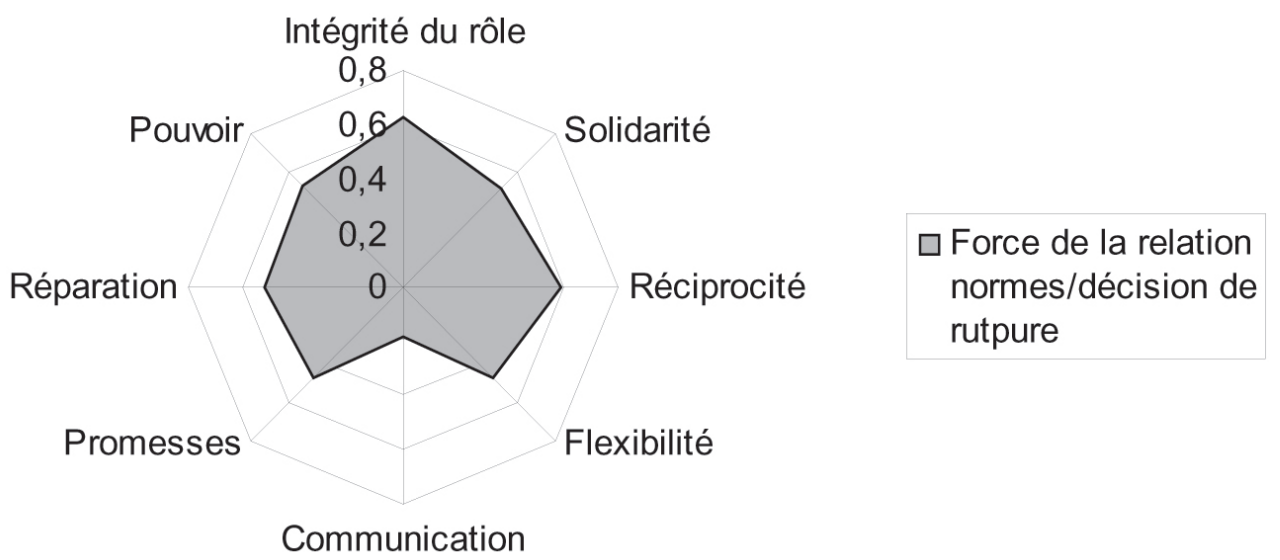

Par ailleurs, les données collectées dans le cadre de l'étude quantitative montrent que certaines normes transactionnelles ont autant d'importance pour la continuité de la relation que les normes relationnelles. Ainsi, sur les huit normes évaluées, quatre ont une influence prépondérante : l'Intégrité du rôle et la Solidarité (normes relationnelles) ainsi que la Réciprocité et le Pouvoir (normes transactionnelles).

Les paragraphes précédents ont permis de mettre en exergue l'existence des normes dans les relations bancaires. À présent, il semble important d’envisager de façon concrète leur rôle et leur valeur dans la performance de l'entreprise.

\subsection{Le rôle de la gestion relationnelle dans la création de valeur}

Charreaux et Wirtz (2006, p. 265) soulignent la nécessité « d’intégrer la dimension cognitive pour apprécier l'impact de la gouvernance sur la création de valeur des entreprises ». Ces auteurs encouragent le développement de grilles de lecture permettant d'apprécier le processus de création dans une perspective cognitive de la gouvernance, susceptible de compléter l'approche disciplinaire traditionnelle. Ainsi, le contrat social proposé par Macneil peut être utilisé, et a été utilisé, comme une grille de lecture spécifique, focalisée sur la création de valeur provenant de la gestion des relations de l'entreprise.

5 L'influence de chacune des normes contractuelles est ici mesurée par un indice de force de la relation, compris entre 0 et 1 . Cet indice permet, au-delà de la significativité de la relation entre deux variables, de se poser la question de l'importance de cette relation. Ainsi, plus la valeur dans la figure est élevée (proche de 1) et donc vers l'extérieur du radar, plus la relation est forte. Plus la valeur dans la figure est faible (proche de 0 ) et donc proche du centre du radar, plus la relation est faible. 
La gouvernance et la création de valeur associée mettent en exergue deux éléments essentiels : l'importance d'une gestion relationnelle comme mécanisme de gouvernance des échanges bancaires et élément central dans le processus de création de valeur (Charreaux, 1997, 1998 ; Wirtz, 2008) et l'accroissement de la valeur partenariale (Dubost, 1996) et également la création de valeur partagée (Porter et Kramer, 2011).

Ces deux éléments concourent à l’amélioration de la performance des entreprises au travers de la gestion relationnelle des échanges.

\subsubsection{UNE GESTION RELATIONNELLE COMME MÉCANISME DE GOUVERNANCE DES ÉCHANGES BANCAIRES}

Dans la lignée de cette position, les résultats des deux études présentées (en finance organisationnelle et en marketing inter-organisationnel) dévoilent que les mécanismes de gouvernance des échanges bancaires s'inscrivent très souvent dans une perspective de gestion intentionnellement relationnelle résultant de la capacité d'action des dirigeants.

Les données collectées dans les deux recherches mettent également en lumière le choix volontaire d'une organisation et d'un fonctionnement relationnels des échanges bancaires de la part des dirigeants de PME.

Ainsi, les données collectées avec un regard « finance organisationnelle » montrent que la proximité relationnelle que les dirigeants entretiennent avec leurs banques n'est pas considérée comme un mode de régulation indépendant, mais comme un mécanisme de gouvernance complémentaire du mécanisme traditionnel de marché. Le rôle des dirigeants est alors essentiel : ils choisissent, initient, impulsent et développent les échanges relationnels en référence aux normes de Solidarité, d'Intégrité de rôle ou de Flexibilité. À titre d'exemple, au regard de la norme de Solidarité, le prix n'est pas le critère essentiel de l'attribution des flux et des crédits.

Le tableau 2 ci-aprés montre, à titre d’exemple, les schémas décisionnels des dirigeants au regard de la norme de Solidarité.

\subsubsection{L'ACCROISSEMENT DE LA VALEUR PARTENARIALE AU REGARD DES NORMES CONTRACTUELLES}

Un très grand nombre de recherches montre l'intérêt pour une entreprise d'établir de véritables partenariats inter-organisationnels. En effet, cette démarche permet généralement d'améliorer les performances et de réaliser des progrès profitant aux deux parties. Comme le rappelle Richomme-Huet (2001), une stratégie relationnelle se fonde sur des relations privilégiées plutôt que sur la concurrence qui devient un facteur de gestion de la relation secondaire par rapport à un " accord scellé de gré à gré et échappant aux règles du marché » (Stratégor, 1991 ; Richomme-Huet, 2001). Ces partenariats permettent à l'entreprise de se procurer des ressources et des compétences sur des marchés, rendus ainsi captifs, reposant sur des accords préférentiels et sur la solidarité. Par exemple, Fenneteau (1990) démontre l'intérêt de mener une coopération durable avec ses fournisseurs plutôt que de continuellement les mettre en concurrence. Cette orientation stratégique permet de limiter le comportement opportuniste des fournisseurs et de stimuler leurs efforts d'innovation et d'investissements. 
Tableau 2. Dimensions de la NORME de solidarité

\begin{tabular}{|c|c|c|c|c|c|}
\hline \multirow{5}{*}{$\begin{array}{l}\text { Raisons du } \\
\text { comportement } \\
\text { solidaire de } \\
\text { l'entreprise }\end{array}$} & $\begin{array}{l}\text { Raison } \\
\text { invoquée }\end{array}$ & Explicitation & \multirow{5}{*}{$\begin{array}{l}\text { Comportement } \\
\text { solidaire de } \\
\text { l'entreprise } \\
\text { (ou préservation } \\
\text { de la relation) }\end{array}$} & Indicateurs & $\begin{array}{l}\text { Mise en évi- } \\
\text { dence empi- } \\
\text { rique }\end{array}$ \\
\hline & $\begin{array}{l}\text { Soutien } \\
\text { passé et } \\
\text { présent de } \\
\text { lentreprise } \\
\text { par la } \\
\text { banque }\end{array}$ & $\begin{array}{l}\text { Le dirigeant est } \\
\text { reconnaissant } \\
\text { d'un comporte- } \\
\text { ment solidaire } \\
\text { antérieur de la } \\
\text { banque }\end{array}$ & & $\begin{array}{l}\text { Partage } \\
\text { des crédits } \\
\text { (investisse- } \\
\text { ments) }\end{array}$ & $\begin{array}{l}\text { Les flux et les } \\
\text { crédits sont } \\
\text { partagés équi- } \\
\text { tablement entre } \\
\text { les banques } \\
\text { de l'entreprise. } \\
\text { Cette démarche } \\
\text { permet de } \\
\text { garantir la } \\
\text { continuité des } \\
\text { relations }\end{array}$ \\
\hline & \multirow{2}{*}{$\begin{array}{l}\text { Soutien } \\
\text { futur } \\
\text { espéré de la } \\
\text { banque }\end{array}$} & \multirow{2}{*}{$\begin{array}{l}\text { Le dirigeant } \\
\text { agit en prévi- } \\
\text { sion d'investis- } \\
\text { sements à venir } \\
\text { ou de besoins } \\
\text { potentiels (ex. : } \\
\text { problème de } \\
\text { trésorerie) }\end{array}$} & & $\begin{array}{l}\text { Avantage } \\
\text { donné aux } \\
\text { banques de } \\
\text { l'entreprise }\end{array}$ & $\begin{array}{l}\text { Lors d'un nou- } \\
\text { veau besoin, } \\
\text { les banques de } \\
\text { l'entreprise sont } \\
\text { prioritaires }\end{array}$ \\
\hline & & & & $\begin{array}{l}\text { Négo- } \\
\text { ciation } \\
\text { prix }\end{array}$ & $\begin{array}{l}\text { La négociation } \\
\text { des prix ne } \\
\text { revient pas à } \\
\text { rechercher sys- } \\
\text { tématiquement } \\
\text { les prix les plus } \\
\text { bas }\end{array}$ \\
\hline & $\begin{array}{l}\text { Réponse à } \\
\text { la demande } \\
\text { des } \\
\text { banques }\end{array}$ & $\begin{array}{l}\text { Les sollicita- } \\
\text { tions régulières } \\
\text { du chargé de } \\
\text { clientèle de la } \\
\text { banque obligent } \\
\text { le dirigeant à } \\
\text { justifier de son } \\
\text { comportement } \\
\text { solidaire }\end{array}$ & & $\begin{array}{l}\text { Placement } \\
\text { des } \\
\text { produits }\end{array}$ & $\begin{array}{l}\text { L'entreprise } \\
\text { accepte des } \\
\text { produits non } \\
\text { essentiels pour } \\
\text { entretenir la } \\
\text { relation }\end{array}$ \\
\hline
\end{tabular}

La littérature en finance et marketing bancaire ainsi que les études réalisées par des banques montrent également l'intérêt des échanges relationnels pour accroitre l'efficacité des interactions tant du point de vue de la banque que de celui de ses clients. Les deux recherches, présentées dans cet article, explicitent ainsi comment la gestion relationnelle des échanges bancaires est créatrice de valeur partenariale pour l'entreprise. Le développement des normes équivaut à la mise en place d'un code de « bonne conduite » bilatéral qui accroît l'efficacité des échanges (cf. Tableau 3). 
TABleau 3. Apports de la gestion RelationNelle D'APrès LeS NORMES RELATIONNELLES

\begin{tabular}{|c|c|}
\hline $\begin{array}{l}\text { Normes } \\
\text { contractuelles } \\
\text { relationnelles }\end{array}$ & Explicitation ou mise en évidence empirique de la création de valeur \\
\hline \multirow{3}{*}{$\begin{array}{l}\text { Intégrité du rôle } \\
\text { du dirigeant/ } \\
\text { communication }\end{array}$} & $\begin{array}{l}\text { La communication du dirigeant est caractérisée par la fréquence des contacts } \\
\text { qui est soutenue et les contacts qui sont recherchés : les contacts les plus } \\
\text { fréquents concernent la vie de l’entreprise, toutes les opportunités de parler } \\
\text { de l'entreprise sont saisies. Les contacts extraordinaires concernent le rachat } \\
\text { d'établissement ou les périodes de fortes difficultés financières. }\end{array}$ \\
\hline & $\begin{array}{l}\text { Le chargé d'affaires doit améliorer sa capacité à identifier les compétences } \\
\text { dans son organisation pour palier ses propres limites, mettre régulièrement à } \\
\text { jour ses connaissances sur les secteurs d'activité de ses clients. Il doit éviter la } \\
\text { politique de l'interlocuteur exclusif afin de mieux gérer les incidences d'une } \\
\text { rotation du personnel en contact. }\end{array}$ \\
\hline & $\begin{array}{l}\text { La valeur partenariale provient de la connaissance réciproque du compor- } \\
\text { tement des acteurs sur le long terme. }\end{array}$ \\
\hline \multirow[t]{2}{*}{ Solidarité } & $\begin{array}{l}\text { Les échanges bancaires s'inscrivent dans la durée ; le comportement soli- } \\
\text { daire de l'entreprise s'explique par le soutien passé et présent des banques de } \\
\text { l'entreprise (continuité de la relation; continuité dans l'approvisionnement } \\
\text { en produits et services nécessaires à l'activité de l'entreprise), mais aussi par le } \\
\text { soutien futur attendu. }\end{array}$ \\
\hline & $\begin{array}{l}\text { La valeur partenariale provient des mécanismes bilatéraux mis en place, } \\
\text { sur le long terme, pour maintenir la relation. }\end{array}$ \\
\hline \multirow{3}{*}{ Flexibilité } & $\begin{array}{l}\text { Ladaptabilité de l'entreprise aux différents fonctionnements des banques, aux } \\
\text { dysfonctionnements et aux périodes critiques (ex. : changements de chargés } \\
\text { d'affaires) répond à l'adaptabilité de la banque (dépassements ponctuels, } \\
\text { déclarés et expliqués [ex. : dépassements de lignes] et rigueur assouplie ponc- } \\
\text { tuellement [ex. : dépassements de lignes]); les échanges bancaires sont alors } \\
\text { harmonieux et sans conflit. }\end{array}$ \\
\hline & $\begin{array}{l}\text { Cette adaptabilité ne passe pas forcément par des démarches informelles, le } \\
\text { formalisme pouvant traduire, aux yeux du client, le sérieux et la rigueur du } \\
\text { chargé d'affaires. }\end{array}$ \\
\hline & $\begin{array}{l}\text { La valeur partenariale provient des mécanismes adaptatifs mis en place, } \\
\text { sur le long terme, pour maintenir la relation. }\end{array}$ \\
\hline
\end{tabular}

Ces résultats rejoignent ceux de Richomme-Huet (2001) qui montre à partir d'une étude de cas sur une coopérative artisanale que la valeur partenariale est accrue par la communication, la coordination, un savoir-faire relationnel spécifique et la réciprocité. Ces éléments renvoient respectivement aux normes de Communication, de Flexibilité, d'Intégrité du rôle et de Réciprocité de la théorie du contrat social. Cette convergence renforce l'idée selon laquelle le contrat social peut être une grille de lecture pertinente de l'efficience des relations inter-organisationnelles. 


\begin{tabular}{|c|c|c|c|}
\hline \multicolumn{2}{|c|}{ Norme Dimensions } & \multirow[b]{2}{*}{$\begin{array}{l}\text { Implication/Chargé d'affaires } \\
\text { Importance de l'empathie du } \\
\text { chargé d'affaires. } \\
\text { Améliorer la capacité du chargé } \\
\text { d'affaires à identifier les com- } \\
\text { pétences dans son organisation } \\
\text { (organigrammes clairs et à jour) } \\
\text { Permettre aux chargés d'affaires } \\
\text { de mettre régulièrement à jour } \\
\text { leur connaissance d'un secteur } \\
\text { d'activité donné : mise à disposi- } \\
\text { tion de fiches sectorielles. }\end{array}$} & \multirow[b]{2}{*}{$\begin{array}{l}\text { Implication/Dirigeant } \\
\text { Le dirigeant choisit, initie, impulse } \\
\text { et développe le mode de gestion des } \\
\text { échanges bancaires, notamment } \\
\text { un mode de gestion relationnel ; le } \\
\text { profil du dirigeant (gestionnaire/ } \\
\text { ingénieur)... et donc la manière de } \\
\text { parler de l’entreprise (activité/domaine } \\
\text { financier) ainsi que sa personnalité } \\
\text { (expérience vécue de la vie de l'entre- } \\
\text { prise/mode de négociation adopté/ } \\
\text { comportement plus ou moins relation- } \\
\text { nel...) influencent le mode de gestion } \\
\text { des échanges choisi et mis en place. }\end{array}$} \\
\hline \multirow{5}{*}{ 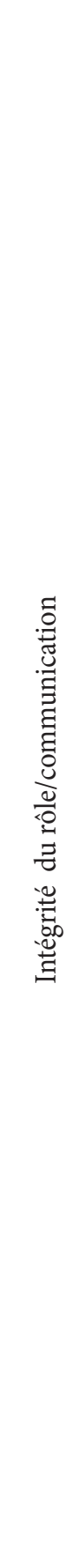 } & $\begin{array}{l}\text { Compétence } \\
\text { du chargé } \\
\text { d'affaires }\end{array}$ & & \\
\hline & $\begin{array}{l}\text { Multiplicité } \\
\text { et niveaux } \\
\text { des contacts } \\
\text { dans les } \\
\text { organisations }\end{array}$ & $\begin{array}{l}\text { Ne pas changer simultanément } \\
\text { les différents interlocuteurs } \\
\text { (dans la banque) d'une relation. } \\
\text { Expliquer à sa clientèle l'intérêt } \\
\text { pour le bon déroulement de la } \\
\text { relation et pour sa continuité } \\
\text { que plusieurs personnes bien } \\
\text { coordonnées soient impliquées } \\
\text { dans la relation chez les deux } \\
\text { partenaires. }\end{array}$ & $\begin{array}{l}\text { Les échanges bancaires ont lieu avec le } \\
\text { dirigeant et le comptable ; le diri- } \\
\text { geant explicite au comptable le mode } \\
\text { de gestion des échanges choisi ; une } \\
\text { forte cohérence des propos et actions } \\
\text { du dirigeant et du comptable est une } \\
\text { condition nécessaire des échanges } \\
\text { relationnels. }\end{array}$ \\
\hline & $\begin{array}{l}\text { Information } \\
\text { sur le } \\
\text { changement } \\
\text { de chargé } \\
\text { d'affaires }\end{array}$ & $\begin{array}{l}\text { Avertir le client du changement } \\
\text { de son chargé d’affaires avant } \\
\text { que le changement n'intervienne } \\
\text { et non pas de façon rétroactive. }\end{array}$ & $\begin{array}{l}\text { Le changement de chargé d’affaires } \\
\text { est vécu comme une période à ne pas } \\
\text { "manquer »: l'intensité, lextensivité } \\
\text { et la proactivité des échanges avec le } \\
\text { nouveau chargé d'affaires sont accen- } \\
\text { tuées en début de relation. }\end{array}$ \\
\hline & $\begin{array}{l}\text { Com- } \\
\text { munication }\end{array}$ & $\begin{array}{l}\text { Repérer les clients qui sont peu } \\
\text { contraints dans la relation et } \\
\text { améliorer la communication. }\end{array}$ & $\begin{array}{l}\text { La communication est essentielle: } \\
\text { la gestion relationnelle implique une } \\
\text { communication intensive, extensive et } \\
\text { proactive de la part de lentreprise. }\end{array}$ \\
\hline & $\begin{array}{l}\text { Fréquence } \\
\text { des ren- } \\
\text { contres }\end{array}$ & $\begin{array}{l}\text { Évaluer régulièrement (ex. : } \\
\text { présentation des bilans) la } \\
\text { fréquence à laquelle le client } \\
\text { souhaite rencontrer son chargé } \\
\text { d’affaires. }\end{array}$ & $\begin{array}{l}\text { La communication est régulière ; le } \\
\text { face à face est régulièrement recher- } \\
\text { ché ; la remise des bilans est un } \\
\text { moment clé où l'entreprise doit com- } \\
\text { muniquer ; tout événement de la vie } \\
\text { de l'entreprise est saisi pour commu- } \\
\text { niquer sur l'entreprise, souvent avant } \\
\text { même que la banque n'ait connais- } \\
\text { sance de l'événement (forte proactivité } \\
\text { valorisée par la banque). }\end{array}$ \\
\hline
\end{tabular}




\begin{tabular}{|c|c|c|c|}
\hline 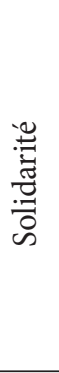 & & $\begin{array}{l}\text { Nécessité pour le chargé d'af- } \\
\text { faires de bien connaitre la rela- } \\
\text { tion de façon à la récompenser } \\
\text { de façon « juste ». Importance } \\
\text { de la mémoire institutionnelle et } \\
\text { de la préparation de la passation } \\
\text { de pouvoir pour assurer un bon } \\
\text { suivi de la relation lors du chan- } \\
\text { gement de chargé d'affaires. }\end{array}$ & $\begin{array}{l}\text { Le dirigeant fait le lien entre les } \\
\text { échanges passés, présents et futurs } \\
\text { avec sa et ses banques; le soutien } \\
\text { passé d'une banque est valorisé dans } \\
\text { les échanges présents; les échanges } \\
\text { présents tiennent compte des échanges } \\
\text { futurs souhaités ; le prix n'est plus alors } \\
\text { la variable essentielle. }\end{array}$ \\
\hline \multirow{4}{*}{ 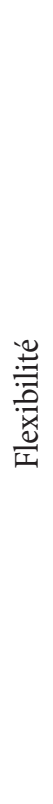 } & $\begin{array}{l}\text { Anticipation } \\
\text { de la } \\
\text { flexibilité }\end{array}$ & $\begin{array}{l}\text { Repérer les clients qui sont } \\
\text { peu contraints dans la relation } \\
\text { et améliorer la flexibilité de la } \\
\text { relation. }\end{array}$ & $\begin{array}{l}\text { Lentreprise (dirigeant et comptable) } \\
\text { identifie les éléments ou opérations } \\
\text { caractérisant le comportement flexible } \\
\text { de la banque et y répond elle-même } \\
\text { par un comportement flexible. }\end{array}$ \\
\hline & $\begin{array}{l}\text { Dispo- } \\
\text { nibilité du } \\
\text { chargé d'af- } \\
\text { faires }\end{array}$ & $\begin{array}{l}\text { Le chargé d’affaires doit être } \\
\text { disponible, c'est-à-dire pou- } \\
\text { voir répondre rapidement aux } \\
\text { demandes des clients. }\end{array}$ & $\begin{array}{l}\text { Les opérations complexes sont repor- } \\
\text { tées, en l'absence du chargé d’affaires, } \\
\text { jusqu'à son retour afin d'éviter tout } \\
\text { problème plus difficile à résoudre. }\end{array}$ \\
\hline & $\begin{array}{l}\text { Caractère } \\
\text { informel } \\
\text { de la relation }\end{array}$ & $\begin{array}{l}\text { Une relation moins formelle } \\
\text { n'est pas forcément une action } \\
\text { pertinente, car le formalisme } \\
\text { traduit le sérieux et la rigueur du } \\
\text { conseiller. }\end{array}$ & \\
\hline & $\begin{array}{l}\text { Multi- } \\
\text { bancarité }\end{array}$ & & $\begin{array}{l}\text { L’entreprise utilise la multibancarité } \\
\text { afin de faciliter les échanges bancaires } \\
\text { (identification de fonctionnements/ } \\
\text { procédures facilitées ou non selon les } \\
\text { banques). }\end{array}$ \\
\hline
\end{tabular}

\subsubsection{VERS UN ENSEMBLE DE RECOMMANDATIONS}

Ainsi, l'analyse des relations bancaires au travers de la grille des normes contractuelles permet de formuler des recommandations précises pour une meilleure gestion des interactions entre le chargé d'affaires et le dirigeant de PME. Le tableau 4, en donne une illustration à partir des normes relationnelles.

Les deux études montrent comment les normes relationnelles peuvent permettre d'identifier, de structurer et de définir le comportement relationnel des clients. Les normes jouent ainsi un rôle clé dans la mise en œuvre de pratiques de gestion au quotidien plus personnalisées.

\section{CONCLUSION : LES PRINCIPAUX ENSEIGNEMENTS ET PROLONGEMENTS POSSIBLES}

La réflexion menée dans cet article montre que le cadre d’analyse proposé par Macneil offre une grille de lecture pertinente de la compréhension des mécanismes (particuliers) de coopération 
et de gouvernance. Lanalyse assortie proposée en reposant sur des travaux issus de champs disciplinaires différents (marketing et finance) montre la relative universalité de ce cadre d’analyse au sein des sciences de gestion. En effet, quelle que soit la discipline d'entrée de l'étude, il ressort que l'analyse des relations interpersonnelles peut être réalisée de façon pertinente au travers de cette grille de lecture. Cette analyse est par ailleurs enrichie par la complémentarité des approches qualitatives et quantitatives au sein de l'analyse assortie proposée permettant de mieux définir et développer les normes et outils de mesure des normes contractuelles de Macneil.

Lanalyse des différentes catégories de normes montre des différences notables : si les normes transactionnelles reposent généralement sur des objets plutôt formalisés (contrat, bilan, procédures, etc.), les normes relationnelles quant à elles renvoient plus à des modes de communication, des règles ou des pratiques plus informelles (culture, valeurs, qualité des échanges, complémentarité, etc.).

Enfin, la présente recherche montre que l'orientation relationnelle d'une entreprise est peu réfléchie et formalisée. Ainsi, si les dirigeants ne parlent généralement pas explicitement d’une gestion relationnelle, les récits des entreprises étudiées montrent en revanche une mise en œuvre opérationnelle de cette gestion relationnelle. De ce fait, même s'il n'y a pas de volonté délibérée de gérer globalement les relations, force est de constater que les organisations intègrent quand même cette préoccupation dans leur gouvernance. Les normes contribuent ainsi de fait à la bonne gouvernance des relations quelles soient formalisées ou seulement des positions de principe.

Subséquemment, le cadre de Macneil est porteur de valeur pour les entreprises à plusieurs titres : il permet aux entreprises (1) de réfléchir sur les moyens mobilisés dans lorganisation, (2) de les formaliser et (3), de travailler à leur combinaison. En effet, le contrat social fait référence à un dispositif de gestion relationnelle reposant sur l'utilisation conjointe d'une définition et d'une combinaison de normes propres à chaque organisation (mécanismes spécifiques) et à l'environnement dans lequel elle évolue (mécanismes non spécifiques ${ }^{6}$ ). Au-delà de son utilité pour réaliser un bilan de la gestion des relations de l'entreprise, les normes contractuelles peuvent également servir de cadre prescriptif.

Dans cette perspective, il serait intéressant de mener une réflexion plus large et novatrice sur la pertinence du cadre de Macneil pour l'analyse de l'ensemble des relations de l'entreprise, et pas seulement des relations inter-organisationnelles classiques. Il ouvre notamment d'intéressantes perspectives dans les champs de la gestion des ressources humaines, de la stratégie et de la gouvernance.

\section{BIBLIOGRAPHIE}

Ambroise L., Maque I. et Prim-Allaz I. (2011), Ian Macneil et la théorie du contrat social : proposition d'un outil d'analyse de la valeur relationnelle pour l'ensemble des domaines de gestion, dans Valeur et outils de gestion, C. Dominguez-Péry (dir.), Paris, Hermès Science Publications-Lavoisier (Organisation et stratégie ; 1), 189-236.

6 Les mécanismes non spécifiques peuvent être par exemple associés à l'environnement légal et règlementaire d'un pays. Ils se situent à un niveau macro, contrairement aux mécanismes spécifiques qui sont à un niveau micro de la gouvernance (Wirtz, 2008). 
Blois K.J. et Ivens B.S. (2006), Measuring relational norms : some methodological issues, European Journal of Marketing, 40, 3-4, 352-365.

Blois K.J. et Ivens B.S. (2007), Methods issues in the measurement of relational norms », Journal of Business Research, 60, 5, 556-565.

Caby J. et Hirigoyen G. (2001), La création de valeur de lentreprise, $2^{e}$ édition, Paris, Economica, $139 \mathrm{p}$.

Chabaud D. et Germain O. (2006), « La réutilisation de données qualitatives en sciences de gestion: un second choix?»,M@n@gement, 9, 3, 199-221.

Charreaux G. (1997), Le Gouvernement des entreprises : théories et faits, Paris, Economica (Recherche en gestion), $570 \mathrm{p}$.

Charreaux G. (1998), Le rôle de la confiance dans les systèmes de gouvernances des entreprises, Économies et sociétés, <http://leg2.u-bourgogne.fr/wp/0980501.pdf>, consulté le 21 octobre 2013.

Charreaux G. et Desbrières P. (1998), Gouvernances des entreprises : valeurs partenariales contre valeurs actionnariales, Finance Contrôle Stratégie, 2, 1, 57-88.

Charreaux G. et Wirtz P. (2006), Gouvernance des entreprises. Nouvelles perspectives, Paris, Economica (Recherche en gestion), $394 \mathrm{p}$.

Duвost N. (1996), Une exemple de stratégie de renforcement des moyennes entreprises (ME) : l'échange relationnel comme moyen de gérer la dépendance économique envers les clients, Revue Internationale PME, 9, 2, 125-142.

DURIF F. (2007), L'impact de la norme - Intégrité du rôle sur les relations d'affaires interentreprises : une étude exploratoire dans le secteur des services financiers, dans les Actes du colloque de l'Association francophone pour le savoir, Québec, Université du Québec à Trois-Rivières, mai.

Durif F. et Perrien J. (2008), Can cognitive mapping enrich our understanding of Macneil's contractual norms? The specific case of role integrity, Journal of Relationship Marketing, 7, 1, 91-120.

Dwyer F.R., Schurr P.H. et Oh S. (1987), Developing buyer-seller relations, Journal of Marketing, 51, 2, 11-28.

Fenneteau H. (1990), Mise en concurrence des fournisseurs ou partenariats ?, Revue Internationale PME, 3, 2, 167-192.

Fink R.C., Hatten K.J. et James W.L. (2010), Time and the salience of Macneil's relational norms, Journal of Targeting, Measurement and Analysis for Marketing, 18, 2, 95-107.

ForD D. (1990), Understanding business markets : interaction, relationships, networks, Londres, Academic Press, 556 p.

Gomez P.-Y. (1994), Qualité et théorie des conventions, Paris, Economica, 282 p.

Heide J.B. et Wathne K.H. (2006), Friends, businesspeople, and relationship roles : a conceptual framework and a research agenda, Journal of Marketing, 70, 3, 90-103.

InStitut DE L'EnTREPRISE (2010), Rapport Annuel 2010, <www.institut-entreprise.fr>, consulté le 21 octobre 2013.

Ivens B.S. et BLois K.J. (2004), Relational exchanges norms in marketing : a critical review of Macneil's contribution, Marketing Theory, 4, 3, 239-263. 
Lamarque E. (2007), Le dirigeant et le banquier, dans Comités exécutifs - Voyage au cour de la dirigeance, Bournois F. et al. (dir.), Paris, Eyrolles-Éditions d'Organisation, 595-600.

Macaulay S. (1963), Non-contractual relations in business : a preliminary study, American Sociological Study, 28, 55-70.

MACNEIL I.R. (1978), Contracts : adjustments of long-term economic relations under classical, neoclassical and relational contract law, Northwestern Law Review, 72, 6, 854-905.

MACNEIL I.R. (1980), The new social contract : an inquiry into modern contractual relations, Londres, Yale University Press, $164 \mathrm{p}$.

MACNEIL I.R. (1981), Economic analysis of contractual relations : its shortfalls and the need for « a rich classificatory apparatus ", Northwestern Law Review, 75, 6, 1018-1063.

MaCneIL I.R. (1983), Values in contract : internal and external, Northwestern Law Review, 78, 2, $340-418$.

Macneil I.R. (2000), Relational contract theory : challenges and queries, Northwestern Law Review, 94, 3, 877-907.

MAQUE I. (2007), Les relations bancaires d'une entreprise : fonctionnement et organisation - Une application à la PME, Thèse de doctorat en sciences de gestion, Université Montesquieu - Bordeaux 4, $479 \mathrm{p}$.

Paulin M. et Ferguson R.J. (2010), Relational norms in interfirm exchanges : from transactional cost economics to the service-dominant logic, Journal of Business-to-Business Marketing, 17, 4, 365-405.

Perrien J., Graf R. et Colombel L. (2005), Le rôle des normes dans lévolution d'une relation, dans Cahier de Recherche, Montréal, ESG-UQAM, 18 p.

Perrien J., Paradis S. et Bantig P.M. (1995), Dissolution of a relationship, the saleforce perception, Industrial Marketing Management, 24, 4, 317-327.

Porter M.E. et Kramer M.R. (2011), The big idea : creating shared value, Harvard Business Review, $80,1,1-17$.

Prim-Allaz I. (2000), Les ruptures de relations de long-terme entre organisations : contributions à létude des déterminants - Une application aux relations banques-PME, Thèse de doctorat en sciences de gestion, Université Paris IX - Dauphine, 401 p.

Richomme-Huet K. (2001), De la cotraitance au management relationnel : le cas d'une coopérative artisanale du secteur du bâtiment, Revue internationale PME, 14, 2, 109-131.

STRATÉGOR (1991), Stratégie, structure, décision, identité : politique générale dentreprise, Paris, InterÉditions, $615 \mathrm{p}$.

Thibaut J.W. et Kelley H.H. (1959), The social psychology of groups, New York, Wiley, 313 p.

Varadarajan P. et Rajaratnam D. (1986), Symbiotic marketing revisited, Journal of Marketing, 50, $1,1-17$.

Whitford W.C. (1985), Ian Macneil's contribution to contracts scholarship, Wisconsin Law Review, 545-561.

Williamson O.E. (1985), The economic institutions of capitalism : firms, markets, relational contracting, New York, Free Press, 468 p. 
WirTz P. (2008), Les meilleures pratiques de gouvernance d'entreprise, Paris, La Découverte (Repères), $121 \mathrm{p}$.

Young J.A., Gilbert F.W. et McIntyre F.S. (1996), An investigation of relationalism across a range of marketing relationships and alliances, Journal of Business Research, 35, 2, 139-151. 